\title{
A Study on the Causes for Child Trafficking using Indeterministic Triangular Fuzzy Cognitive Maps
}

\author{
A.Praveen Prakash \\ Department of Mathematics \\ Hindustan University \\ Padur, Chennai-603103
}

\author{
M.P.Kannan \\ Department of Mathematics \\ Velammal,Mat, $\mathrm{Hr}, \mathrm{Sec}, \mathrm{School}$ \\ Mogappair, Chennai-600037
}

\author{
J.Esther Jerlin \\ Department of Mathematics \\ Hindustan University \\ Padur, Chennai-603103
}

\begin{abstract}
"Dad who sold three babies caught during fourth deal is the headline of the news in Indian Express dated 31.05.2014. The culprit named Praveen (37) confessed to the crime and is in police custody. His wife Manju was already rescued and admitted to a Home. News of this kind have become a regular feature. Child trafficking takes place all over the world, and is indeed prevalent in India. There have been reports from many areas about the increase of trafficking taking place in India. This paper analyses the causes of Child Trafficking and ranking the causes using the newly proposed Indeterministic Triangular Fuzzy Cognitive Model . This model has an advantage of ranking the causes by the calculated membership value also showing indeterminacy relation between the nodes obtained from the experts. This paper has four sections. Section one deals with the overall existing problem of child trafficking. Section two deals with the description of the model. Section three gives the study and analysis of the problem using the prescribed model. Section four gives the conclusion and suggestions based on the study.
\end{abstract}

\section{Keywords}

FCMs, NCMs, TrNCMs, Hidden pattern, fixed point, Child Trafficking.

\section{INTRODUCTION}

According to the US State Department, there are approximately 600,000 to 820,000 people trafficked a year across international borders, and up to $50 \%$ of those are children. This is definitely seen as a growing issue in Asia, with many children that are and continue to be trafficked for many reasons as well as being exploited. It acts as a form of modern - day slavery. It is defined as the recruitment, harboring, transportation, obtaining a person by means of force, fraud or coercion for the purpose of a commercial sex act or labor services. It continues to be the second largest criminal industry in the world.

The newly proposed model helps in ranking the attributes in a refined manner. It involves the basis of FCM, NCM and TrFCM. FCM deals with the on-off state of the attributes. NCM deals with the indeterminacy condition between the attributes. TrFCM ranks the attributes based on the membership value obtained from Traverage. TrFCM relies on giving interrelationship degree value between the attributes though indeterminacy is seen between them. But this new model have an advantage of using inderterminate condition by denoting I so that the membership value obtained from TrNCM average shows the minute difference in ranking than TrFCM. [1, 2, 4]

\section{PRELIMINARIES}

\subsection{Triangular Fuzzy number}

It is a fuzzy number represented with three points as follows. The membership function defined as

$$
\mu_{A}(x)= \begin{cases}0, & \text { for } x<a_{1} \\ \frac{x-a_{1}}{a_{2}-a_{1}} & \text { for } a_{1} \leq x \leq a_{2} \\ \frac{a_{3}-x}{a_{3}-a_{2}} & \text { for } a_{2} \leq x \leq a_{3} \\ 0 & \text { for } x>a_{3}\end{cases}
$$

2.2 Operation of Triangular Fuzzy Number The following are the four operations that can be performed on triangular fuzzy numbers: Let $A=\left(a_{1}, a_{2}, a_{3}\right)$ and $B=\left(b_{1}, b_{2}, b_{3}\right)$ then,

(i) Addition (+): $A+B=\left(a_{1}+b_{1}, a_{2}+b_{2}, a_{3}+b_{3}\right)$

(ii) Subtraction (-): $A+B=\left(a_{1}-b_{1}, a_{2}-b_{2}, a_{3}-b_{3}\right)$

(iii) Multiplication $(\otimes)$ :

(a) $k \otimes A=\left(k a_{1}, k a_{2}, k a_{3}\right), k \in R, k \geq 0$

(b) $A \otimes b=\left(a_{1} b_{1}, a_{2} b_{2}, c_{1} c_{2}\right), a_{1} \geq 0, a_{2} \geq 0$

(iv) Division $(\varnothing)$ :

$$
\begin{gathered}
(A)^{-1}=\left(a_{1}, b_{1}, c_{1}\right)^{-1} \cong\left(1 / c_{1}, 1 / b_{1}, 1 / a_{1}\right), a_{1}>0 \\
A \varnothing B \cong\left(a_{1} / c_{2}, b_{1} / b_{2}, c_{1} / a_{2}\right), a_{1} \geq 0, a_{2}>0
\end{gathered}
$$

\subsection{Degrees of the Triangular Fuzzy Number}

The linguistic values of the triangular fuzzy numbers are 
Table 1 : Linguistic Values of the Triangular Fuzzy Numbers

\begin{tabular}{|c|c|}
\hline Very Low & $(0,0,0.25)$ \\
\hline Low & $(0,0.25,0.50)$ \\
\hline Medium & $(0.25,0.50,0.75)$ \\
\hline High & $(0.50,0.75,1)$ \\
\hline Very High & $(0.75,1,1)$ \\
\hline
\end{tabular}

DEFINITION 2.3.1: In the neutrosophic logic every logical variable $\mathrm{x}$ is described by an ordered triple $\mathrm{x}=(\mathrm{T}, \mathrm{I}$, $\mathrm{F}$ ) where $\mathrm{T}$ is the degree of truth, $\mathrm{F}$ is the degree of false and I level of indeterminacy.

DEFINITION 2.3.2: A Neutrosophic Cognitive Map (NCM) is a neutrosophic directed graph with concepts like policies, events etc. as nodes and causalities or indeterminates as edges. It represents the causal relationship between concepts.

Let $\mathrm{C}_{1}, \mathrm{C}_{2}, \ldots \mathrm{C}_{\mathrm{n}}$ denote $\mathrm{n}$ nodes, further we assume each node is a neutrosophic vector from neutrosophic vector space V. So a node $C_{i}$ will be represented by $\left(x_{1}, \ldots\right.$, $x_{n}$ ) where $x_{k}$ 's are zero or one or I (I is the indeterminate) and $\mathrm{x}_{\mathrm{k}}=1$ means that the node $\mathrm{C}_{\mathrm{k}}$ is in the on state and $\mathrm{x}_{\mathrm{k}}=0$ means the node is in the off state and $x_{k}=I$ means the nodes state is an indeterminate at that time or in that situation. Let $\mathrm{C}_{\mathrm{i}}$ and $\mathrm{C}_{\mathrm{j}}$ denote the two nodes of the NCM. The directed edge from $C_{i}$ to $C_{j}$ denotes the causality of $\mathrm{C}_{\mathrm{i}}$ on $\mathrm{C}_{\mathrm{j}}$ called connections. Every edge in the NCM is weighted with a number in the set $\{-1,0,1, I\}$. Let $e_{i j}$ be the weight of the directed edge $\mathrm{C}_{\mathrm{i}} \mathrm{C}_{\mathrm{j}}, \mathrm{e}_{\mathrm{ij}} \in\{-1,0,1, \mathrm{I}\} . \mathrm{e}_{\mathrm{ij}}=0$ if $C_{i}$ does not have any effect on $C_{j}, e_{i j}=1$ if increase (or decrease) in $C_{i}$ causes increase (or decreases) in $C_{i}, e_{i j}$ $=-1$ if increase (or decrease) in $\mathrm{C}_{\mathrm{i}}$ causes decrease (or increase) in $C_{j} \cdot e_{i j}=I$ if the relation or effect of $C_{i}$ on $C_{j}$ is an indeterminate. [3]

\subsection{Basic Definitions of Indeterministic Triangular Fuzzy Cognitive Maps (TrNCMs)}

DEFINITION 2.4.1: Triangular NCMs with edge weights or causalities from the set $\{-1,0,1, I\}$ are called simple Triangular NCMs.

DEFINITION 2.4.2: A TrNCM is a directed graph with concepts like policies, events etc, as nodes and causalities as edges, It represents causal relationships between concepts.

DEFINITION 2.4.3: Consider the nodes/concepts ${ }_{\mathrm{Tr}} \mathrm{C}_{1}$, ${ }_{\mathrm{Tr}} \mathrm{C} 2, \ldots,{ }_{\mathrm{Tr}} \mathrm{Cn}$ of the Triangular NCM. Suppose the directed graph is drawn using edge weight ${ }_{\mathrm{T}_{\mathrm{r}}} \mathrm{eij} \in\{-1,0,1, \mathrm{I}\}$. The triangular neutrosophic matrix $\mathrm{M}$ be defined by $\operatorname{TrN}(\mathrm{M})=$ $\left.{ }_{{ }_{\mathrm{Tr}}} \mathrm{eij}\right)$ where ${ }_{\mathrm{Tr}} \mathrm{eij}$ is the triangular neutrosophic weight of the directed edge ${ }_{T r} C_{i T r} C_{j}$. $\operatorname{TrN}(M)$ is called the adjacency matrix of Triangular Neutrosophic Cognitive Maps, also known as the connection matrix of the TrNCM. It is important to note that all matrices associated with a TrNCM are always square matrices with diagonal entries as zero.
DEFINITION 2.4.4: Let $\operatorname{TrC}_{1}, \operatorname{TrC}_{2}, \ldots, \operatorname{TrCn}$ be the nodes of an TrNCM. $A=\left(a_{1}, a_{2}, \ldots, a_{n}\right)$ where Treije $\{-1,0,1, I\}$. A is called the instantaneous state vector and it denotes the on-off position of the node at an instant.

Instantaneous vector

$a_{i}=0$ if $a_{i}$ is off (has no effect)

$a_{i}=1 \quad$ if $a_{i}$ is on, (has effect)

$\mathrm{a}_{\mathrm{i}}=\mathrm{I} \quad$ if $\mathrm{a}_{\mathrm{i}}$ is indeterminate, (effect cannot be determined) where $\mathrm{i}=1,2, \ldots, \mathrm{n}$.

DEFINITION 2.4.5: Let $\operatorname{TrC}_{1}, \operatorname{TrC}_{2}, \ldots, \operatorname{TrC}_{\mathrm{n}}$ be the nodes of TrNCM. Let be the edges of the TrNCM $(i \neq j)$. Then the edges form a directed cycle. A TrNCM is said to be cyclic if it possesses a directed cycle. A TrNCM is said to be acyclic if it does not possess any directed cycle.

DEFINITION 2.4.6: A TrNCM is said to be cyclic it is said to have a feedback.

DEFINITION 2.4.7: When there is a feedback in a TrNCM, i.e., when the causal relations flow through a cycle in a revolutionary way, the TrNCM is called a dynamical system.

DEFINITION 2.4.8: Let $\overrightarrow{{ }_{T r} C_{1 T r} C_{2}},{ }_{T r} C_{2 T r} C_{3}, \overrightarrow{{ }_{T r} C_{3 T r} C_{4}}, \ldots \overrightarrow{{ }_{T r} C_{n-1 T r} C_{n}}$ be a cycle. When $\mathrm{TrCi}$ is switched $\mathrm{ON}$ and if the causality flows through the triangular edges of a cycle and if it again causes $\mathrm{Ci}$, we say that the dynamical system goes round and round. This is true for any triangular node $\operatorname{TrCi}$ for $\mathrm{i}=1,2, \ldots, \mathrm{n}$. The equilibrium state for this dynamical system is called the hidden pattern.

DEFINITION 2.4.9: If the equilibrium state of a dynamical system is a unique state vector, then it is called a fixed point. Consider a $\operatorname{TrNCM}$ with $\operatorname{TrC}_{1}, \operatorname{TrC}_{2}, \ldots, \operatorname{TrCn}$ as nodes. For example let us start the dynamical system by switching on $\operatorname{TrC}_{1}$.Let us assume that the TrNCM settles down with $\operatorname{TrC} 1$ and $\operatorname{TrCn} \mathrm{ON}$ i.e., in the state vector remains as $(1,0,0, \ldots, 0)$ is called fixed point.

DEFINITION 2.4.10: If the TrNCM settles down with a state vector repeating in the form $\mathrm{A}_{1} \rightarrow \mathrm{A}_{2} \rightarrow \ldots \rightarrow \mathrm{A}_{\mathrm{i}} \rightarrow \mathrm{A}_{1}$ then this equilibrium is called a limit cycle.

\subsection{Algorithm used to manipulate TrNCM}

Step 1: Consider the attributes given by the experts for the problem as $\operatorname{TrC}_{1}, \operatorname{TrC}_{2}, \ldots . \operatorname{TrC}_{\mathrm{n}}$. According to the feedback given by the expert directed graph is drawn.

Step 2: Let $\operatorname{TrN}(\mathrm{M})$ denotes the connection matrix with $\{-$ $1,0,1, \mathrm{I}\}$ as causalities.

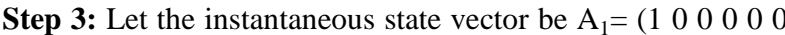
$\left.\begin{array}{llll}0 & 0 & 0 & 0\end{array}\right)$. It is passed into $\operatorname{TrN}(\mathrm{M})$. The on state vector $\operatorname{TrN}(\mathrm{M})_{\text {avgweight }}$ is obtained.

Step 4: Maximum membership value is threshold as 1 and other as 0 and I. Consider the threshold and updated vector as $\mathrm{A}_{2}$.

Step 5: $A_{2}$ is passed into $\operatorname{TrN}(M)$ the above said calculation is performed, threshold and updated until the same fixed point. [5] 


\section{ANALYSIS OF THE PROBLEM}

According to the experts opinion ten attributes were taken for the analysis of the problem.[7,9]

${ }_{T r} C_{1}$ - Entertainment

${ }_{\operatorname{Tr}} C_{2}$ - Illiteracy

${ }_{T r} C_{3}$ - Unemployment

${ }_{T r} C_{4}$ - Poverty

${ }_{T r} C_{5}$-Sexual Exploitation
${ }_{T r} C_{6}$ - Bonded Labour

${ }_{T r} C_{7}$ - Political Uprising

${ }_{T r} C_{8}$ - Illegal Activities

${ }_{T r} C_{9}$ - Social Factor

${ }_{T r} C_{10}$ - High profit at low risk

The following matrix $\operatorname{TrN}(\mathrm{M})$ gives the linguistic connection matrix with inderterminacy.

\begin{tabular}{|c|c|c|c|c|c|c|c|c|c|c|}
\hline & ${ }_{I r} C_{1}$ & ${ }_{T r} C_{2}$ & ${ }_{I r} C_{3}$ & ${ }_{I r} C_{4}$ & ${ }_{I r} C_{5}$ & ${ }_{I r} C_{6}$ & ${ }_{{ }_{T r}} C_{7}$ & ${ }_{{ }_{I r}} C_{8}$ & ${ }_{T r} C_{9}$ & ${ }_{T r} C_{10}$ \\
\hline${ }_{I r} C_{1}$ & 0 & $V H$ & $L$ & $M$ & $H$ & $H$ & $M$ & $V L$ & $I$ & $V L$ \\
\hline${ }_{T r} C_{2}$ & $M$ & 0 & $V H$ & $H$ & $M$ & $H$ & $M$ & $L$ & $V L$ & $L$ \\
\hline${ }_{T r} C_{3}$ & $V H$ & $M$ & 0 & $H$ & $M$ & $L$ & $V L$ & $M$ & $H$ & $L$ \\
\hline${ }_{T r} C_{4}$ & $L$ & $M$ & $L$ & 0 & $H$ & $H$ & $M$ & 1 & $V L$ & $V H$ \\
\hline${ }_{T r} C_{5}$ & $V L$ & $L$ & $L$ & $H$ & 0 & $M$ & $L$ & $V H$ & $I$ & $H$ \\
\hline${ }_{7 r} C_{6}$ & $V L$ & $L$ & $H$ & $M$ & $V H$ & 0 & $H$ & $L$ & $V L$ & $H$ \\
\hline${ }_{7 r} C_{7}$ & $M$ & $H$ & $L$ & $M$ & $V L$ & $H$ & 0 & $M$ & $V H$ & $H$ \\
\hline${ }_{I r} C_{8}$ & $V L$ & $M$ & $H$ & $H$ & $M$ & $L$ & $V L$ & 0 & $L$ & $V H$ \\
\hline${ }_{T r} C_{9}$ & $H$ & $H$ & $L$ & $V L$ & $M$ & $L$ & $V L$ & $V H$ & 0 & $M$ \\
\hline$C_{10}$ & $V L$ & $M$ & $L$ & $H$ & $H$ & $M$ & $L$ & VH & $V L$ & 0 \\
\hline
\end{tabular}

Fig 1: Linguistic Connection Matrix with Inderterminacy

$\operatorname{TrN}(\mathrm{M})_{\text {average weight }}$ matrix is given below.

\begin{tabular}{|c|c|c|c|c|c|c|c|c|c|c|}
\hline & ${ }_{I r} C_{1}$ & ${ }_{I r} C_{2}$ & ${ }_{I r} C_{3}$ & ${ }_{{ }_{T r}} C_{4}$ & ${ }_{{ }_{I r}} C_{5}$ & ${ }_{I r} C_{6}$ & ${ }_{I r} C_{7}$ & ${ }_{I r} C_{8}$ & ${ }_{I r} C_{9}$ & ${ }_{T r} C_{10}$ \\
\hline${ }_{{ }_{I r}} C_{1}$ & 0 & 0.91 & 0.25 & 0.5 & 0.75 & 0.75 & 0.5 & 0.08 & $I$ & 0.08 \\
\hline${ }_{{ }_{T r}} C_{2}$ & 0.5 & 0 & 0.91 & 0.75 & 0.5 & 0.75 & 0.5 & 0.25 & 0.08 & .25 \\
\hline${ }_{{ }_{T}} C_{3}$ & 0.91 & 0.5 & 0 & 0.75 & 0.5 & 0.25 & 0.08 & 0.5 & 0.75 & 0.25 \\
\hline${ }_{I r} C_{4}$ & 0.25 & 0.5 & 0.25 & 0 & 0.75 & 0.75 & 0.5 & $I$ & 0.08 & 0.91 \\
\hline${ }_{I_{r}} C_{5}$ & 0.08 & 0.25 & 0.25 & 0.75 & 0 & 0.5 & 0.25 & 0.91 & $I$ & 0.75 \\
\hline${ }_{I_{r}} C_{6}$ & 0.08 & 0.25 & 0.75 & 0.5 & 0.91 & 0 & 0.75 & 0.25 & 0.08 & 0.75 \\
\hline${ }_{I r} C_{7}$ & 0.5 & 0.75 & 0.25 & 0.5 & 0.08 & 0.75 & 0 & 0.5 & 0.91 & 0.75 \\
\hline${ }_{I r} C_{8}$ & 0.08 & 0.5 & 0.75 & 0.75 & 0.5 & 0.25 & 0.08 & 0 & 0.25 & 0.91 \\
\hline${ }_{I r} C_{9}$ & 0.75 & 0.75 & 0.25 & 0.08 & 0.5 & 0.25 & 0.08 & 0.91 & 0 & 0.5 \\
\hline${ }_{T r} C_{10}$ & 0.08 & 0.5 & 0.25 & 0.75 & 0.75 & 0.5 & 0.25 & 0.91 & 0.08 & 0 \\
\hline
\end{tabular}

Fig 2: $\operatorname{TrN}(M)_{\text {average weight }}$ Matrix 
International Journal of Computer Applications (0975 - 8887)

Volume 103 - No.11, October 2014

Mathematical Sciences International Research Journal, ISSN:2278-8697, ISBN:978-93-84124-03-8, Vol. 3, Issue 1, March 2014.

[8] A.Praveen Prakash, N.Lakshmipathy and J.Esther Jerlin, "Problems of Housemaids in Chennai City A Study Using Combined Fuzzy Cognitive Maps" (CFCMs), International Association of Engineers, ISBN: 978-98819252-7-5, ISSN: 2078-0958, ISSN-2078-0966, Vol.IJuly 2014.

[9] A.Praveen Prakash , M.P Kannan and Esther Jerlin.J, “A
Study on the causes for Child Trafficking using Combined Fuzzy Cognitive Maps" (CFCMs), ELSEVIER, ISBN: 978-93-51072-61-4, September 2014.

[10] Child Trafficking in India in Wikipedia

[11] A.Rajkumar, A.Victor Devadoss, " A study on Miracles through Holy Bible using new Triangular Neutrosophic Cognitive Maps (TRNCMs)", IJCA, Issue-4, Vol-4, JulyAugust-2014. 